\title{
Diseño de una estrategia de control difuso aplicada al proceso de ultracongelación de alimentos
}

\author{
Design of fuzzy control strategy applied to food process deep freeze
}

\author{
Rafael J. García ${ }^{1} \quad$ Angel D. Pinto ${ }^{2} \quad$ José Eduardo Rengel $^{3}$ \\ Juan M. Torres ${ }^{2} \quad$ Jaime A. González ${ }^{4} \quad$ Nelson A. Pérez ${ }^{4 *}$ \\ Recibido 4 de diciembre de 2015, Aceptado 24 de mayo de 2016 \\ Received: December 4, 2015 Accepted: May 24, 2016
}

\begin{abstract}
RESUMEN
El control de la temperatura de los alimentos mediante la utilización de un equipo de congelación criogénico se torna complicado cuando se emplean técnicas de control clásico tipo PID (Proporcional + Integral + Derivativo), debido al fuerte acoplamiento entre las variables y las no linealidades propias del sistema. Actualmente, en la mayoría de las plantas procesadoras de alimentos que utilizan túneles de congelación criogénico, el control es llevado a cabo por medio del ajuste manual de los valores de consigna o setpoint de los lazos de control de velocidad y temperatura del túnel de congelación, lo que trae como consecuencia desviaciones en la salida del proceso, derivadas principalmente de la diferencia de criterios existentes entre los operadores y de la velocidad de respuesta de estos últimos ante variaciones en las condiciones del proceso. En este trabajo se tratan separadamente los lazos de control de velocidad y temperatura, se reconfiguran y entonan los lazos existentes, para luego desarrollar un controlador difuso tipo Takagi-Sugeno que permite ajustar el valor de consigna de la temperatura del túnel de congelación en función al producto y las condiciones de entrada del alimento al proceso. El comportamiento del sistema se comprueba mediante la simulación del mismo, verificándose que se mantiene la temperatura del producto en $-18{ }^{\circ} \mathrm{C}$, pese a que fue sometido a cambios en la masa de producto a enfriar (de $200 \mathrm{~kg}$ a $220 \mathrm{~kg}$ ) y en el flujo másico del producto (de $650 \mathrm{~kg} / \mathrm{h} \mathrm{a} 700 \mathrm{~kg} / \mathrm{h}$ ).
\end{abstract}

Palabras clave: Congelación de alimentos, controlador PID, identificación de sistemas, control difuso, controlador Takagi-Sugeno.

\section{ABSTRACT}

The control of the temperature of foods using cryogenic freezing equipment, becomes complicated when techniques of classical control as PID are used (Proportional + Integral + Derivative), due to the strong coupling between the variables and the non-linearities of the system. Currently, in most food processing plants that use tunnels of cryogenic freezing, the control is carried out through manual adjustments of set-points of control loops of the speed and temperature of the freezing tunnel, which results in deviations

1 Departamento de Electricidad. Universidad de Oriente. Av. Argimiro Gabaldón, Puerto La Cruz, Venezuela. E-mail: ecem118@hotmail.com

2 Escuela de Ingeniería de Sistemas. Universidad del Sinú. Carrera 1w. № 38-153. Barrio Juan XIII. Montería, Colombia. E-mail: anpima1@hotmail.com; jtorrestovio@yahoo.com

3 Departamento de Mecánica. Universidad de Oriente. Av. Argimiro Gabaldón. Puerto La Cruz, Venezuela. E-mail: jrengel.anz@udo.edu.ve; rengel66@gmail.com

4 Escuela de Ingeniería Eléctrica. Universidad de Los Andes. Av. Alberto Carnevali, Núcleo "Pedro Rincón Gutiérrez". Edificio Facultad de Ingeniería. Mérida, Venezuela. E-mail: gcjaime@gmail.com; perezn@ula.ve; nelsonp90@hotmail.com

* Autor de correspondencia 
in the process output, derived mainly from the difference of criteria existing between operators and the response speed of them to variations in the process conditions. In this paper, we treat separately the control loops of speed and temperature, the current control loops are reconfigured and are intoned, and finally, is developed a Takagi-Sugeno fuzzy controller to adjust the set-point of the temperature of the freezing tunnel, in function of the product and the conditions of entry of food into the process. The behavior of the system is tested by its simulation, verifying that the system maintains the product temperature at $-18^{\circ} \mathrm{C}$, regardless of changes in the product mass to cool (from $200 \mathrm{~kg}$ to $220 \mathrm{~kg}$ ) and in the mass flow of the product (from $650 \mathrm{~kg} / \mathrm{h}$ to $700 \mathrm{~kg} / \mathrm{h}$ ).

Keywords: Food freezing, PID controller, systems identification, fuzzy control, Takagi-Sugeno controller.

\section{INTRODUCCIÓN}

La ultracongelación, también conocida como sobrecongelación o congelación rápida, es el proceso mediante el cual los alimentos son congelados en el menor tiempo posible ( 2 horas como máximo, dependiendo del tipo de producto), conllevando a que los mismos puedan conservar la mayor parte de sus cualidades, ya que su estructura celular se ve poco afectada por el menor tamaño de los cristales de hielo que se forman tanto en el interior de la célula como en los espacios entre células, durante el proceso en cuestión [1].

Se puede decir que la ultracongelación ocurre cuando el alimento ha alcanzado los $-18{ }^{\circ} \mathrm{C}$ en el centro térmico del mismo [2]. Por tanto, el alimento debe ser almacenado a una temperatura igual o inferior a la misma.

La conversión del agua contenida en el alimento o producto, desde el estado líquido al sólido, que tiene lugar durante el proceso de congelación, produce cambios significativos en las propiedades de dicho alimento. Entre tales propiedades se tiene la densidad, conductividad térmica, entalpía, difusividad térmica, calor latente y calor específico; propiedades que a su vez dependen de factores composición de los productos, contenido de humedad, temperatura y constituyentes de los mismos alimentos [3].

El agua, las proteínas, los lípidos, los carbohidratos, la ceniza y la fibra son los compuestos constituyentes que más afectan las propiedades térmicas de los alimentos; esto debido a su variabilidad y características, las que están estrechamente relacionadas con la temperatura.

En la Tabla 1 se muestran variaciones de algunas de las propiedades físicas de los alimentos, en función de la temperatura, $T$, según algunos de sus compuestos [4].

Tabla 1. Ecuaciones de cálculo de algunas de las propiedades térmicas de los alimentos.

\begin{tabular}{|c|c|l|}
\hline $\begin{array}{c}\text { Propiedad } \\
\text { fisicotérmica }\end{array}$ & Componente & \multicolumn{1}{c|}{ Ecuación } \\
\hline \multirow{3}{*}{$\begin{array}{c}K_{\mathrm{c}} \\
\left(\mathrm{W} / \mathrm{m}^{\circ} \mathrm{C}\right)\end{array}$} & Agua & $\begin{array}{l}0,571-1,76 \times 10^{-3} \\
\boldsymbol{T}-6,70 \times 10^{-7} \boldsymbol{T}^{2}\end{array}$ \\
\cline { 2 - 3 } & Lípidos & $\begin{array}{l}0,180+2,76 \times 10^{-3} \\
\boldsymbol{T}-1,77 \times 10^{-7} \boldsymbol{T}^{2}\end{array}$ \\
\cline { 2 - 3 } & Proteínas & $\begin{array}{l}0,178+1,19 \times 10^{-3} \\
\boldsymbol{T}-2,71 \times 10^{-6} \boldsymbol{T}^{2}\end{array}$ \\
\hline \multirow{3}{\rho}{$\begin{array}{c}\rho \\
\left(\mathrm{Kg} / \mathrm{m}^{3}\right)\end{array}$} & Agua & $\begin{array}{l}997,18+3,14 \times 10^{-3} \\
\boldsymbol{T}-3,75 \times 10^{-3} \boldsymbol{T}^{2}\end{array}$ \\
\cline { 2 - 3 } & Lípidos & $925,59-0,417 \boldsymbol{T}$ \\
\cline { 2 - 3 } & Proteínas & $1329,9-0,518 \boldsymbol{T}$ \\
\hline
\end{tabular}

$K_{c}$ : Coeficiente de conductividad térmica.

$\rho$ : Densidad del producto.

Actualmente resulta relativamente simple realizar el proceso de ultracongelación de alimentos, gracias a la gran potencia frigorífica que poseen los sistemas de congelación basados en nitrógeno líquido, utilizados en túneles criogénicos, los que están conformados por los dos (2) bloques mostrados en las Figuras 1a y 1b: a) una banda transportadora de velocidad variable, que es la encargada de trasladar el producto desde la entrada del túnel de congelación hasta el final del mismo; b) un sistema de congelación encargado de someter el alimento a temperaturas que pueden oscilar entre los $-20{ }^{\circ} \mathrm{C}$ y $-90{ }^{\circ} \mathrm{C}$.

La congelación criogénica es una tecnología que ha ganado popularidad debido a su relativo bajo costo de instalación y mejora en la calidad del alimento congelado. Sin embargo, el alto costo de los fluidos 
criogénicos hace que las principales aplicaciones de ese método estén dirigidas hacia el congelamiento de productos con alto valor agregado, en el que la calidad del alimento compensa el costo de obtención. Cuando la variabilidad en la carga de calor de entrada y/o las características del producto es alta, la economía llega a ser desfavorable debido a sobreenfriamiento o subenfriamiento, lo cual provoca un uso excesivo de fluido criogénico o reducción del rendimiento [5]. Por tanto, es preciso un buen mecanismo de control que minimice las pérdidas debidas a sobreenfriamiento o subenfriamiento y al mismo tiempo mantenga el rendimiento requerido. En ese sentido, se han propuesto esquemas de control que buscan dar respuesta a la mencionada problemática, como los descritos en [6-9].

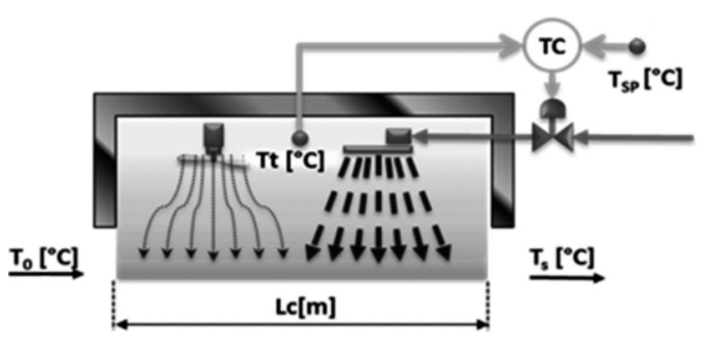

(a)

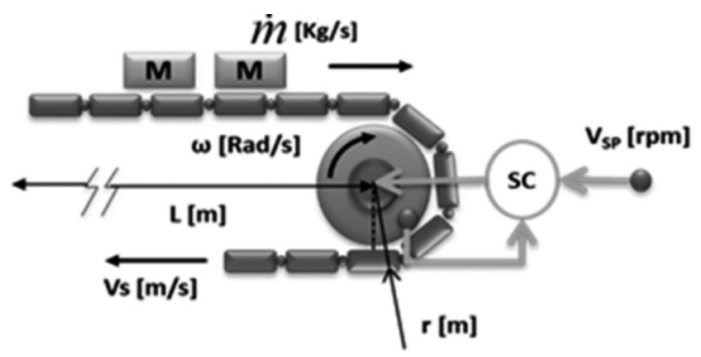

(b)

Figura 1. a) Túnel de congelación; b) Banda transportadora.

En la Figura 1 los parámetros representados son:

$\boldsymbol{T}_{\mathbf{0}}$ : Temperatura inicial del alimento $\left[{ }^{\circ} \mathrm{C}\right]$.

$\boldsymbol{T}_{\mathrm{t}}$ : Temperatura del túnel de congelación $\left[{ }^{\circ} \mathrm{C}\right]$.

$\boldsymbol{T}_{\mathrm{s}}$ : Temperatura de salida del alimento $\left[{ }^{\circ} \mathrm{C}\right]$.

$\boldsymbol{L}_{\mathbf{c}}$ : Longitud del túnel de congelamiento [m].

$\boldsymbol{T}_{\mathrm{sp}}$ : Temperatura del valor de consigna $\left[{ }^{\circ} \mathrm{C}\right]$.

M: Masa $(\mathrm{kg})$.

$\boldsymbol{m}$ : Flujo másico de la masa $[\mathrm{kg} / \mathrm{s}]$.
$\boldsymbol{V}_{\mathbf{s}}:$ Velocidad de salida $[\mathrm{m} / \mathrm{s}]$.

$\boldsymbol{V}_{\text {sp }}$ : Velocidad del valor de consigna $[\mathrm{m} / \mathrm{s}]$.

$\omega$ : Velocidad angular del motor de la banda transportadora [rad/seg].

$r$ : Radio del cilindro de tracción de la banda transportadora $[\mathrm{m}]$.

$\boldsymbol{L}: \quad$ Distancia $[\mathrm{m}]$.

En el presente trabajo se diseñó una estrategia de control mediante la combinación de técnicas de control clásico y lógica difusa, para llevar a cabo el acoplamiento de los lazos de control de flujo y temperatura de un proceso de congelación de pescado (churrascos de atún) que utiliza un equipo de congelación criogénico de aspersión con inyección de nitrógeno líquido dentro de un túnel de alimentación continua. Se propone un controlador PID (Proporcional + Integral + Derivativo) para la velocidad de giro del rodillo de la banda transportadora del alimento y un controlador fuzzy del tipo Takagi-Sugeno que fija el valor de consigna del controlador PI (Proporcional + Integral) usado para regular la temperatura en el túnel de congelamiento. Se recurrió a un proceso de identificación para desarrollar los modelos matemáticos necesarios, logrando, de esta manera, superar las dificultades que en este sentido ofrece la dinámica no lineal del congelamiento criogénico causado por el cambio de fase y la dinámica térmica entre los productos congelados y el túnel [10].

\section{TIEMPO DE CONGELAMIENTO}

La principal ventaja del congelamiento con nitrógeno líquido es el reducido tiempo que el producto requiere en el equipo de enfriamiento. Para controlar ese proceso de congelamiento se necesita determinar el tiempo requerido para que la temperatura en el centro térmico del producto alcance $\operatorname{los}-18^{\circ} \mathrm{C}$. El procedimiento en cuestión, para una pieza de alimento de forma irregular, en un ambiente a temperatura constante representa una gran dificultad [11-12]. En la práctica, para tal fin se recurre a una serie de suposiciones que permitan obtener un modelo simplificado del proceso de congelación, el que se lleva a cabo en tres (3) etapas: preenfriamiento, congelación y atemperado o subenfriamiento [13-14] tal como puede verse en la Figura 2, en la que $T_{\mathrm{o}}, T \mathrm{c}$ y $T \mathrm{a}$ representan las temperaturas inicial del producto, de congelación y de atemperado, respectivamente; 
mientras que $t_{\mathrm{p}}, t_{\mathrm{c}} \mathrm{y} t_{\mathrm{a}}$ se refieren a los tiempos en los que estabilizan las etapas de preenfriamiento, congelación y atemperado o sub-enfriamiento, respectivamente.

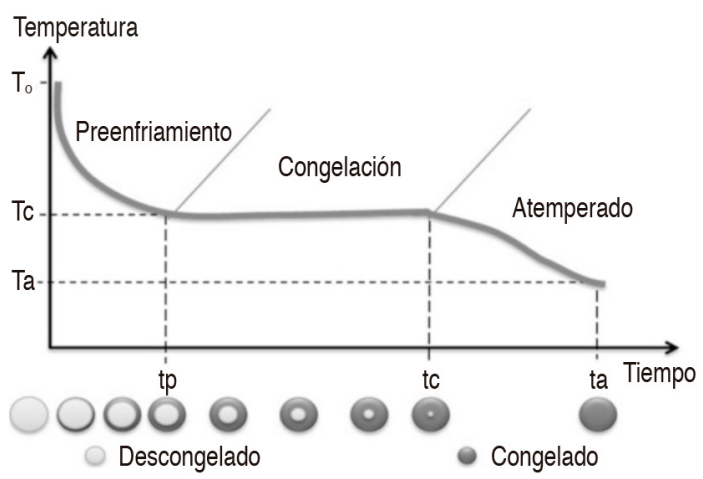

Figura 2. Etapas de la congelación de los alimentos.

A pesar de que existen diversos métodos que se usan para determinar el tiempo de congelamiento [15-17], muchos de los estudios relacionados con la determinación de dicho parámetro hacen referencia principalmente a la ecuación desarrollada por Plank, que permite obtener rápidamente un estimado suficientemente preciso del referido tiempo para uso en ingeniería [3-4].

Por tanto, el tiempo de congelación, $t_{\mathrm{c}}$, del alimento viene dado por:

$$
t_{C}=\frac{\rho \lambda}{T_{t}-T_{o}}\left(\frac{P d}{h_{s}}+\frac{R d^{2}}{k_{c}}\right)
$$

Donde:

$\lambda$ : Calor latente de cristalización $[\mathrm{kJ} / \mathrm{kg}]$.

$\boldsymbol{P}, \boldsymbol{R}$ : Constantes que dependen de las dimensiones $\mathrm{y}$ forma del producto.

$d$ : Espesor del alimento [m].

$\boldsymbol{h}_{\mathrm{s}}$ : Coeficiente convectivo de transferencia de calor $\left[\mathrm{W} /{ }^{\circ} \mathrm{C} \mathrm{m}^{2}\right]$.

\section{IDENTIFICACIÓN DE SISTEMAS}

Con el fin de construir el modelo del controlador difuso objeto del presente trabajo es necesario identificar las variables medidas del proceso, es decir, las entradas (variables de control), salidas (variables controladas) y posibles perturbaciones.

\section{Identificación de sistemas a lazo abierto}

Para obtener de la información dinámica del proceso se excita el mismo y se registra su entrada y salida como tal. En función de los arrojados se pueden aplicar algunas técnicas de identificación experimental, como los métodos basados en la curva de reacción del proceso ante una respuesta escalón o modelos autorregresivos [18]. Tales métodos son de lazo abierto, en los que el controlador puede estar o no instalado (en este último caso, este operaría de modo "manual" durante la prueba) y en base a la respuesta del sistema se puede obtener los parámetros que servirán para representar el modelo del proceso. Para ello se recurre, por ejemplo, al "Método de la tangente de Ziegler y Nichols" [19], "Método de dos puntos de Alfaro" [20], "Método modificado de Smith" [21], entre otros. La aplicación de esos métodos a su vez permitirá obtener un modelo simplificado del sistema, que puede ser de primer orden más tiempo "muerto" o de segundo orden más tiempo "muerto".

Por su parte, un modelo autoregresivo tipo ARX con entrada Exógena (Auto Regressive with eXogenous input), es un modelo discreto lineal en el que la salida en el instante de muestreo " $n$ " se obtiene a partir de valores anteriores de salida y entrada (en n-1, $\mathrm{n}-2$, etc.). Adicionalmente, incorpora un ruido blanco [22]. El modelo como tal se obtiene mediante el cálculo por regresión lineal multivariable de parámetros de ajuste, de manera tal que la diferencia cuadrática entre los valores reales y estimados por el modelo sea mínima.

En el presente trabajo, se usó un modelo ARX, ya que, además de su relativa simplicidad, produjo un excelente ajuste de los datos de entrada-salida registrados al aplicar una entrada escalón al proceso en estudio.

\section{DISEÑO DE CONTROLADORES}

El diseño de controladores se basa en el conjunto de requerimientos necesarios para realizar tareas específicas del proceso. Tales requerimientos, que representan las especificaciones de desempeño del sistema, son la precisión, estabilidad relativa y velocidad de respuesta [18].

Para un desempeño satisfactorio del sistema, el primer paso a ser dado es la realización de solo un ajuste de la ganancia proporcional a lazo abierto. Empero, 
en la mayoría de los casos prácticos ese ajuste por sí solo no proporciona una suficiente alteración del comportamiento del sistema que haga posible cumplir las especificaciones dadas, por lo que se hace necesario modificar la dinámica del sistema.

\section{Método de la ganancia última}

También desarrollado por Ziegler y Nichols [18], se emplea para ajustar el controlador a una curva de respuesta en lazo cerrado, considerando una ganancia "A" amortiguada a razón de 1/4 (Figura 3a) [18]. El método consiste en determinar la ganancia de un controlador de tipo proporcional, que produzca una respuesta de oscilaciones sostenidas, es decir, oscilaciones con amplitud constante, como ilustra la Figura 3b [18]. El período de dicha señal es llamado " $\mathrm{Pu}$ ".

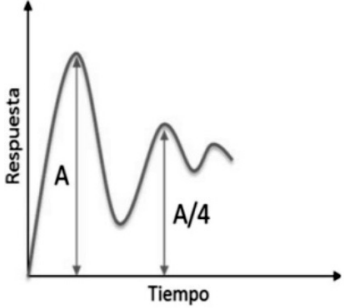

(a)

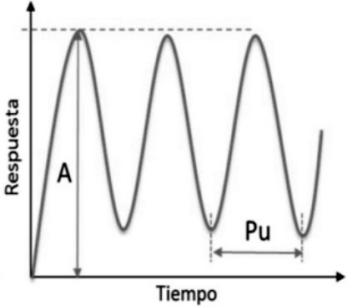

(b)
Figura 3. Curvas de respuestas del sistema a lazo cerrado.

\section{CONTROL DIFUSO}

La técnica de lógica difusa o lógica fuzzy, permite extraer conclusiones cuantitativas a partir de esquemas difusos, vagos o ambiguos propios del razonamiento cualitativo de los humanos. Ese tipo de razonamiento lógico, conformado por observaciones (antecedentes) y reglas cualitativas (base de conocimiento) es aprovechado para incorporar "inteligencia", por ejemplo, en sistemas de control y automatización de procesos industriales [23].

\section{Estructura de un controlador difuso}

El principio básico de un modelo basado en lógica difusa es el conjunto de reglas heurísticas, cuyas variables de entrada y salida, ambas lingüísticas, son representadas mediante conjuntos difusos.

Un controlador difuso está compuesto básicamente por los cuatro (4) bloques operacionales que se muestran en la Figura 4 [24], con " $u$ " y " $y$ " siendo la entrada y salida, respectivamente.

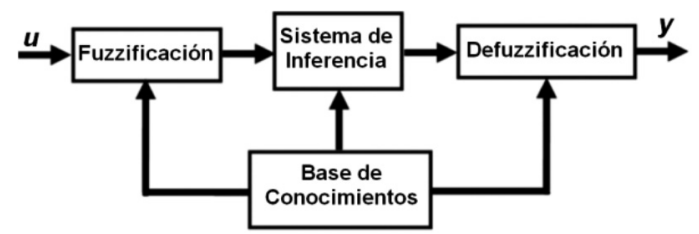

Figura 4. Estructura general de un controlador difuso.

La fuzzificación convierte las variables físicas de entrada del sistema en variables difusas mediante las funciones de pertenencia. Aquí se definen los rangos de variación de las variables de entrada, así como los conjuntos difusos asociados con sus respectivas funciones de pertenencia.

Por su parte, en la defuzzificación, las variables difusas obtenidas del sistema de inferencia son convertidas en variables de reales de salida. Para ello, se suele emplear uno de los siguientes métodos matemáticos [25]: método del máximo, método del centroide o centro de área y método de la media difusa ponderada.

La base de conocimientos o de datos define las reglas lingüísticas del control y la manipulación de la información difusa referente a las funciones de pertenencia de los conjuntos difusos.

Finalmente, el sistema de inferencia se apoya en las reglas del controlador y la inferencia difusa, para calcular las salidas difusas asociadas a las variables reales de salida. En tal sentido, a saber se tienen dos (2) dos tipos de controladores, los que se caracterizan de acuerdo a la forma en que se definen las salidas difusas. Estos son los denominados Mamdani [26] y Takagi-Sugeno [27].

El control difuso tipo Mamdani se caracteriza por la siguiente forma general de sus reglas difusas:

$$
\mathrm{Si} \text { “ } u 1 \text { ” es } A_{\mathrm{i}} \mathrm{y} \text { “ } u 2 \text { ” es } B_{\mathrm{i}} \text {, entonces “y” es } C_{\mathrm{i}}
$$

Es decir, la salida de dicho controlador es un conjunto difuso, por lo que será necesaria una etapa de defuzzificación para obtener un valor discreto en la salida del controlador. 
Por su parte, el control difuso tipo Takagi-Sugeno está caracterizado por la siguiente forma de reglas difusas:

$\mathrm{Si}$ “ $u 1$ ” es $A_{\mathrm{i}} \mathrm{y}$ " $u 2$ ” es $B_{\mathrm{i}}$, entonces $\mathrm{y}=\mathrm{f}(u 1, u 2)$

Lo que significa que la salida del controlador TakagiSugeno no necesitará una etapa de defuzzificación, debido a que se obtiene directamente un valor discreto.

\section{DISEÑO DEL CONTROLADOR DIFUSO PARA EL PROCESO DE ULTRACONGELACIÓN DE ALIMENTOS}

Para el diseño del controlador objeto del presente trabajo se procedió a ejecutar las siguientes etapas:

\section{Etapa 1: Identificación del sistema}

Tal como se ilustra la Figura 1, el sistema de congelación de alimentos basado en un equipo de congelación criogénico, está conformado por un lazo de control de velocidad de banda transportadora y un lazo de control de temperatura del túnel de congelación; ambos lazos tratados de manera separada en el presente trabajo.
Lazo de control de velocidad:

El sistema de control de velocidad de la banda transportadora del equipo de congelación criogénica está basado en un lazo de control PID encargado de regular la velocidad de rotación del cilindro de tracción de la banda transportadora.

Se realizó una simulación de prueba a lazo abierto, para determinar el modelo de representación de la planta, según los métodos descritos anteriormente. Adicionalmente, se utilizó la herramienta de identificación de sistemas que posee el software LabView [28], para obtener el modelo autorregresivo y comparar la respuesta de cada modelo en comparación con los datos reales tomados del sistema, tal como muestra la Figura 5.

De la Figura 5, se observa que los modelos de primer orden más tiempo "muerto" ofrecen una desviación considerable con respecto a la respuesta real, mientras que para el caso de los modelos de segundo orden, si bien ofrecen una menor desviación, su respuesta también difiere del comportamiento real del sistema. En este sentido, el modelo ARX, determinado con el auxilio de la herramienta de LabView SITK (System Identification ToolKit), es

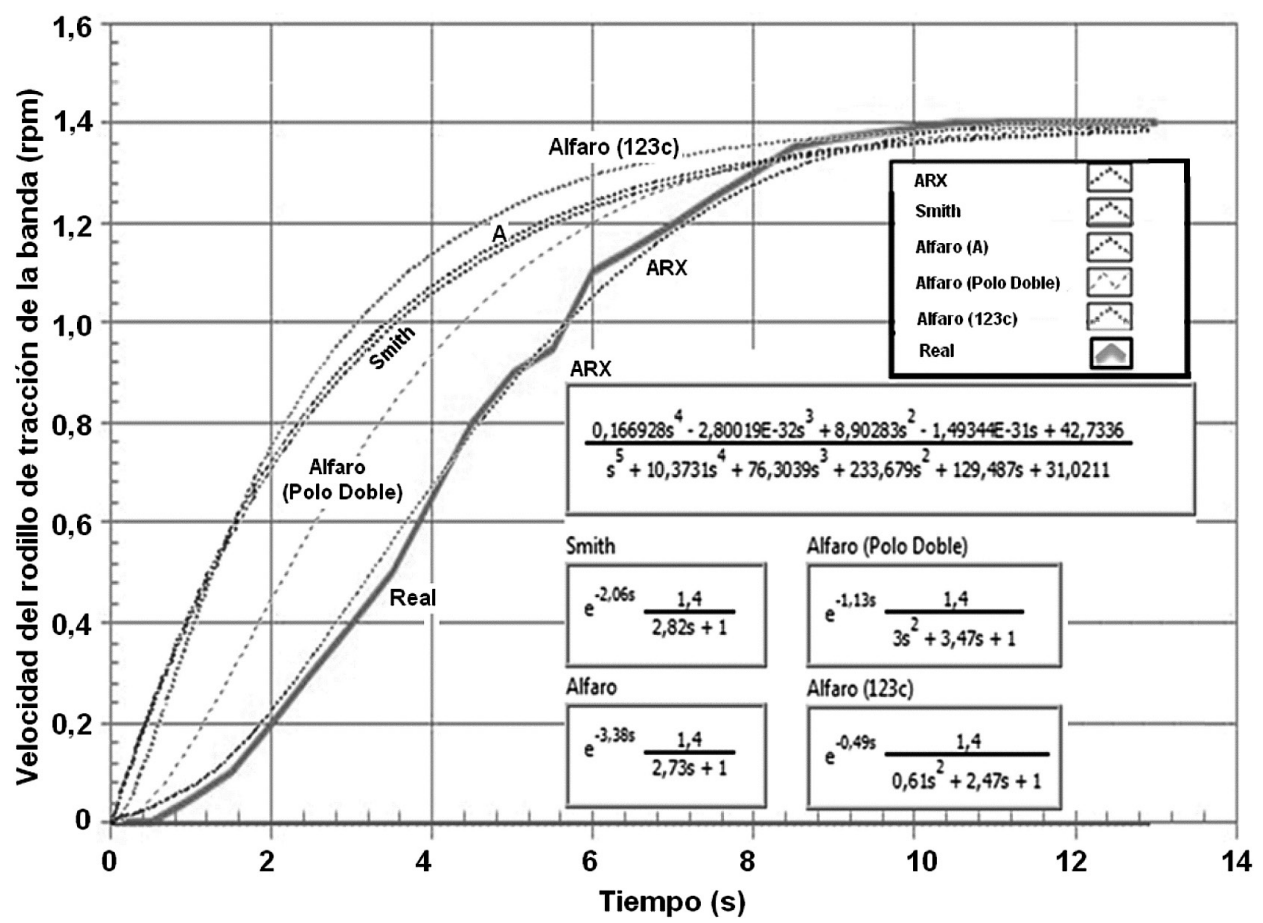

Figura 5. Curvas comparativas de los modelos de representación del lazo abierto de velocidad. 
el que mejor representa al proceso de velocidad de tracción de la banda transportadora, quedando su función de transferencia definida por:

$$
G_{P}(S)=\frac{0,167 S^{4}-2,32 \times 10^{-32} S^{3}+8,90 S^{2}-1,24 \times 10^{-31} S+42,73}{S^{5}+10,37 S^{4}+76,30 S^{3}+233,68 S^{2}+129,49 S+31,02}
$$

Una vez conocido el modelo se procede a determinar los parámetros de entonación del lazo de control de velocidad. Para ello, se recurre a método de la ganancia última de Ziegler y Nichols, obteniéndose los parámetros de ajuste para un control del tipo PID, que ofreció una respuesta con 29,5 segundos de tiempo de asentamiento y un sobrepico inferior al $25 \%$.

\section{Lazo de control de temperatura:}

El funcionamiento del lazo de control de la temperatura del equipo de congelación criogénica se basa en el ajuste de la temperatura del túnel de congelación, mediante la apertura de la válvula de flujo del nitrógeno líquido en función del valor consigna de un controlador PI, tal como se mostró en la Figura 1.

Para realizar la correcta entonación de este lazo de control, se utilizó el mismo procedimiento utilizado para el control de la velocidad, resultando el modelo del proceso térmico del túnel de congelación definido por:

$G_{P}(S)=\frac{0,016 S^{4}-3,30 \times 10^{-34} S^{3}+0,002 S^{2}-4,39 \times 10^{-36} S+2,54 \times 10^{-5}}{S^{5}+0,70 S^{4}+0,18 S^{3}+0,02 S^{2}+0,001 S+1,94 \times 10^{-5}}$

Los parámetros de entonación del lazo de control de temperatura también se estimaron mediante el método de la ganancia última. En este caso, los parámetros ajustados para el controlador tipo PI garantizaron una respuesta con 375 segundos de tiempo de asentamiento y un sobrepico inferior al $20 \%$.

\section{Modelado del proceso de congelación:}

Para determinar el valor de la temperatura final $\left(T_{\mathrm{S}} \mathrm{o}\right.$ $T_{\mathrm{F}}$ ) del alimento, en función al tiempo de exposición o congelación $\left(t_{\mathrm{c}}\right)$, la temperatura del túnel de congelación $\left(T_{\mathrm{T}}\right)$ y la temperatura inicial del alimento $\left(T_{\mathrm{o}}\right.$ o $T_{\mathrm{I}}$ ), se usó como base la ecuación (1), para lo que se tomaron en cuenta las siguientes premisas:

- El calor latente de cristalización $(\lambda)$, también conocido como variación de la entalpía $(\Delta H)$, será determinado en función de la diferencia existente entre la entalpia del alimento a la temperatura final $\left[H_{\mathrm{F}}=\mathrm{h}\left(T_{\mathrm{F}}\right)\right]$ y la entalpia inicial del alimento $\left[H_{\mathrm{I}}=\mathrm{h}\left(T_{\mathrm{I}}\right)\right]$; es decir:

$$
\Delta H=H_{F}-H_{I}=h\left(T_{F}\right)-h\left(T_{I}\right)
$$

- Se considerará que la temperatura del túnel de congelación es uniforme e igual a $T_{\mathrm{T}}$, por medio del largo del túnel de congelación criogénico $\left(L_{\mathrm{c}}\right.$ o $\left.L_{\mathrm{T}}\right)$.

- Las variables serán los valores de la densidad del alimento $(\rho)$ y el coeficiente de conductividad térmica $\left(K_{\mathrm{c}}\right)$, cuyo cálculo estará relacionado con el valor de la temperatura según las ecuaciones de la Tabla 1.

- Se considerará que el espesor del alimento $(d)$ se mantiene constante durante el tiempo de exposición al frío $\left(t_{\mathrm{c}}\right)$.

En base a las premisas anteriores, la temperatura final que alcanzará el alimento se calculará en función al proceso de congelación para una placa infinita, el que se modeló mediante el despeje del valor de la entalpía final de la ecuación (1), como sigue:

$$
h\left(T_{F}\right)=h\left(T_{I}\right)+\frac{t_{c}}{\rho k}\left(T_{T}-T_{I}\right)
$$

En donde $t_{\mathrm{c}}$ se puede determinar por la relación existente entre la velocidad del rodillo de tracción de la banda transportadora $(\omega)$, el radio del rodillo $(r)$ y el largo del túnel de congelación $\left(L_{\mathrm{T}}\right)$, mediante:

$$
t_{c}=\frac{L_{T}}{\omega r}
$$

Por su parte, comparando de las ecuaciones (1) y (7) y asumiendo que el alimento es empacado entre dos placas expuestas al aire, es decir, "P" igual a $1 / 2$ y "R" igual a $1 / 8$ [3], se obtiene que $K$ está relacionado con el espesor " $d$ ", el coeficiente superficial $\left(h_{\mathrm{s}}\right)$ y el coeficiente de conductividad térmica $\left(K_{\mathrm{c}}\right)$, de la siguiente manera:

$$
K=\frac{d}{2 h_{s}}+\frac{d^{2}}{8 K_{c}}
$$

Una vez modelado el proceso de congelación del alimento se procedió a realizar la validación del modelo en relación a los datos reales, los que fueron tomados con un registrador de temperatura (logger). La comparación en cuestión puede verse en la Figura 6. 


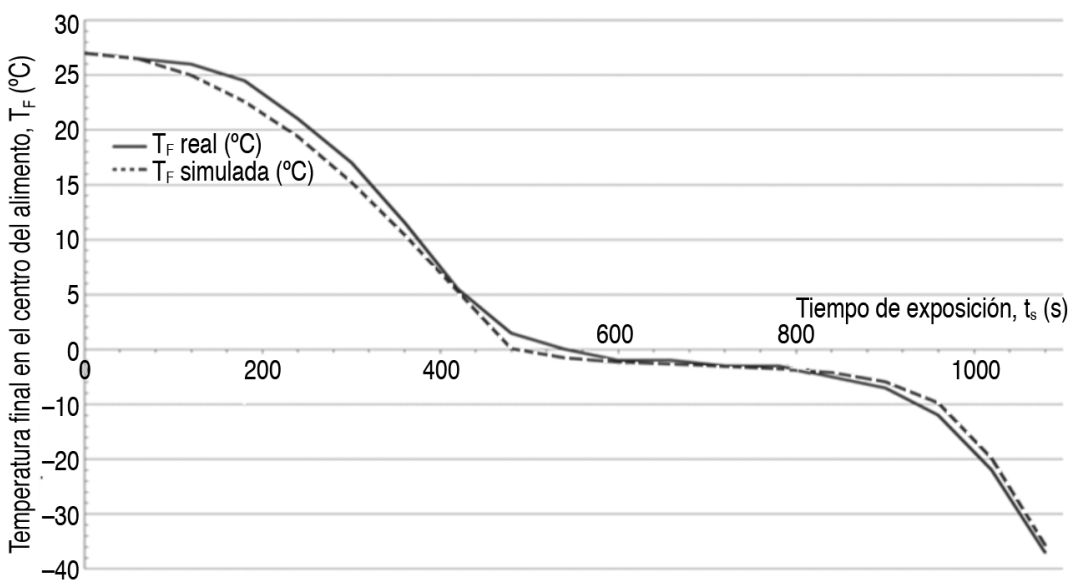

Figura 6. Comparación de la temperatura $T_{\mathrm{F}}$ real vs. temperatura $T_{\mathrm{F}}$ simulada.

Dada la similitud entre ambas respuestas mostradas en la Figura 6, se puede considerar como válido el modelo desarrollado.

\section{Etapa 2: Propuesta de sistema}

\section{Control de flujo másico}

Por lo general, en el proceso de ultracongelamiento de alimentos el operador del equipo fija el valor de la consigna en el panel de control que regula la velocidad de la banda transportadora, en función al flujo de producto deseado,

Por tanto, considerando que la alimentación del producto se realiza de manera manual, es evidente que resulta imposible llevar un correcto control del flujo másico del producto, lo que resulta en pérdidas de la productividad de la línea, así como el desperdicio de la capacidad frigorífica del equipo.

Para solventar dicha problemática, la actual propuesta consiste en modificar el lazo de control mediante la inclusión de un sistema de pesaje continuo, para obtener la relación entre la velocidad de la banda transportadora y la masa colocada en ella, logrando así determinar el flujo másico del proceso, como se ilustra en la Figura 7.

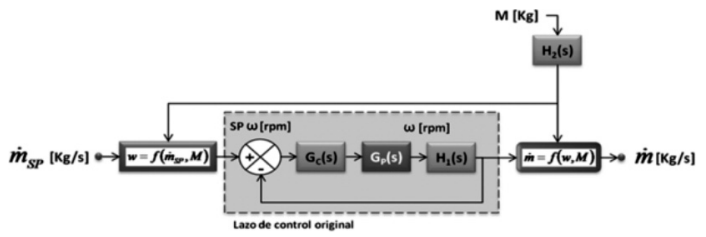

Figura 7. Sistema de control de flujo másico propuesto.

\section{Control de la temperatura de congelación:}

En el sistema a optimizar, el control se logra mediante el ajuste manual del valor de consigna de la temperatura del túnel en función del producto a ser procesado, velocidad de la banda transportadora $(\omega)$ y temperatura que el alimento posee a la entrada del túnel $\left(\mathrm{T}_{\mathrm{I}}\right)$.

En ese proceso de control, el valor de consigna es fijado por el operador, en base a los siguientes criterios:

- La temperatura de funcionamiento debe ser lo más alta posible, con el fin de evitar un consumo de nitrógeno excesivo.

- El controlador de temperatura actúa como un regulador PI.

- Los ajustes de los valores de consigna están orientados a lograr que el alimento alcance al menos $-18^{\circ} \mathrm{C}$ a la salida del túnel de congelación.

Es importante resaltar que la revisión de la temperatura a la salida del túnel la realiza el operador del equipo, quien, al verificar que esta no cumple con la especificación, procede a ajustar el valor de consigna del lazo de control del túnel en base a las premisas arriba mencionadas. Ello redunda en un retardo considerable en la toma de decisión (para el cambio de la consigna o setpoint), lo que ocasiona desviaciones a la salida del proceso de congelación del alimento.

Para superar tales deficiencias, se propone una estrategia de control que centre su funcionamiento 
en la estimación de la temperatura a la cual deberá estar el túnel de congelación $\left(T_{\mathrm{T}}\right)$ para que, en función del tipo de producto a ser procesado, la temperatura inicial $\left(T_{\mathrm{I}}\right)$ y la velocidad del rodillo de la banda transportadora $(\omega)$, se logre alcanzar una temperatura final de $-18^{\circ} \mathrm{C}$ o inferior. Para tal cometido, las variables descritas anteriormente $\left(T_{\mathrm{i}}\right.$, $\omega$ y $T_{\mathrm{T}}$ ) servirán de información crucial para que, mediante una lógica de control difusa, se pueda obtener un valor de consigna para el lazo de control de temperatura del túnel y así lograr, mediante esta, un mejor desempeño comparado al que se tiene con un operador. Para ello, se requerirá realizar algunas modificaciones al equipo actual, que permitan extender las posibilidades de controlar el proceso de congelación de manera automatizada, tal y como se muestra en la Figura 8.

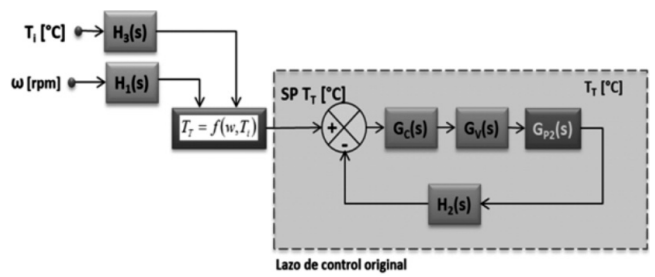

Figura 8. Sistema de control de temperatura propuesto.

\section{Etapa 3: Diseño del control difuso}

El proceso de diseño del controlador se enfoca en la determinación del valor que deberá tener la temperatura del túnel, en función del producto a ser congelado, el valor de la temperatura inicial del mismo y el tiempo que dicho alimento será expuesto dentro del túnel criogénico, de manera que se pueda obtener una temperatura de salida predeterminada al final del proceso de congelación.

Siendo así, se establecen como variables de entrada la temperatura inicial del alimento $\left(T_{\mathrm{I}}\right)$ y la velocidad del rodillo de tracción de la banda transportadora $(\omega)$, mientras que la variable de salida definirá el valor de consigna para el lazo de control de la temperatura del túnel de congelación criogénica $\left(T_{\mathrm{T}}\right)$.

Para el caso de las entradas, se definieron los conjuntos difusos para cada variable (6 para $T_{\mathrm{I}} \mathrm{y}$ 11 para $\omega$ ); mientras que para la variable de salida $\left(T_{\mathrm{T}}\right)$, se definieron siete (7) conjuntos difusos, como lo ilustra la Figura 9.

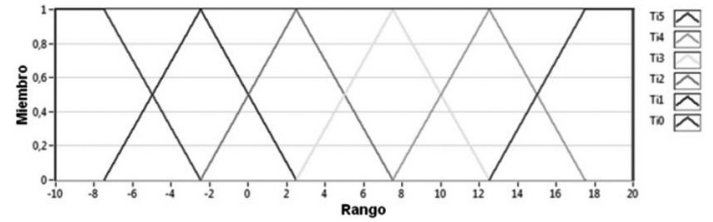

(a)

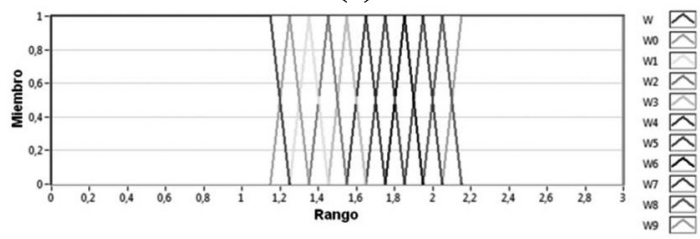

(b)

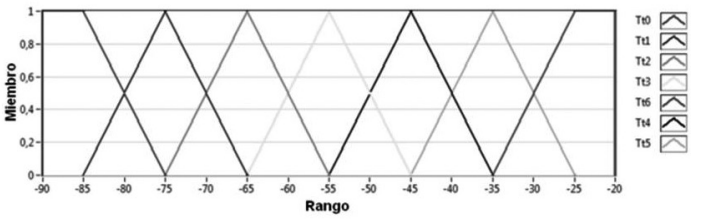

(c)

Figura 9. Variables de entrada y salida del control difuso tipo Mamdani propuesto: a) $T_{\mathrm{I}}$; b) $\omega$; c) $T_{\mathrm{T}}$.

Para la definición de los conjuntos difusos se utilizó el modelo de congelación de alimentos dado por la ecuación (7), con el fin de determinar los valores de $T_{\mathrm{T}}$ en función del par $\left(\omega, T_{\mathrm{I}}\right)$, necesarios para que $T_{\mathrm{F}}$ sea aproximadamente $18{ }^{\circ} \mathrm{C}$. En la Tabla 2 se resumen dicho valores, para el caso específico del producto "churrasco de atún".

Ahora bien, para el escenario considerado en el presente trabajo, se definió el conjunto de reglas basado en un controlador difuso tipo Mamdani. Tomando en cuenta los datos de la Tabla 2, se desarrolló una matriz con la definición de las reglas de inferencia señaladas en la Tabla 3.

Es oportuno resaltar que, como última parte del desarrollo del controlador difuso se seleccionó el proceso de defuzzificación mediante el método del centroide, debido principalmente a su practicidad durante la implementación y su eficiencia.

\section{Validación del controlador mamdani:}

Para verificar la precisión de la respuesta de la salida del controlador, se procedió a determinar, en términos del error relativo y en base a la combinación 
Tabla 2. Valores de $T_{\mathrm{T}}$ en función a $\omega$ y $T_{\mathrm{I}}$ (para el producto “churrasco de atún”).

\begin{tabular}{|c|c|c|c|c|c|c|c|c|c|c|c|c|c|c|}
\hline & \multicolumn{13}{|c|}{ Temperatura inicial, $T_{i}\left({ }^{\circ} \mathrm{C}\right)$} \\
\hline & & 20,0 & 17,5 & 15,0 & 12,5 & 10,0 & 7,50 & 5,00 & 2,50 & 0 & -2.50 & $-5,00$ & $-7,50$ & $-10,0$ \\
\hline \multirow{10}{*}{$\begin{array}{l}\frac{\pi}{0} \\
\frac{0}{0} \\
\frac{\pi}{0} \\
\frac{0}{0} \\
\frac{0}{0} \\
\frac{0}{0}\end{array}$} & 1,2 & $-31,0$ & $-30,5$ & $-30,0$ & $-29,5$ & $-29,0$ & $-28,5$ & $-28,0$ & $-27,5$ & $-27,0$ & $-20,0$ & $-20,0$ & $-20,0$ & $-20,0$ \\
\hline & 1,3 & $-35,5$ & $-35,0$ & $-34,5$ & $-34,0$ & $-33,5$ & $-33,0$ & $-32,0$ & $-31,5$ & $-31,0$ & $-20,0$ & $-20,0$ & $-20,0$ & $-20,0$ \\
\hline & 1,4 & $-40,5$ & $-40,0$ & $-39,5$ & $-38,5$ & $-38,0$ & $-37,5$ & $-36,5$ & $-35,5$ & $-35,0$ & $-21,5$ & $-20,0$ & $-20,0$ & $-20,0$ \\
\hline & 1,5 & $-46,0$ & $-45,5$ & $-44,5$ & $-44,0$ & $-43,0$ & $-42,0$ & $-41,0$ & $-40,0$ & $-39,5$ & $-23,0$ & $-20,0$ & $-20,0$ & $-20,0$ \\
\hline & 1,6 & $-52,0$ & $-51,5$ & $-50,5$ & $-49,5$ & $-48,5$ & $-47,5$ & $-46,5$ & $-45,0$ & $-44,0$ & $-25,0$ & $-20,5$ & $-20,0$ & $-20,0$ \\
\hline & 1,7 & $-58,5$ & $-57,5$ & $-56,0$ & $-55,0$ & $-54,0$ & $-53,0$ & $-51,5$ & $-50,5$ & $-49,0$ & $-26,5$ & $-21,5$ & $-20,5$ & $-20,0$ \\
\hline & 1,8 & $-65,5$ & $-64,0$ & $-63,0$ & $-61,5$ & $-60,0$ & $-59,0$ & $-57,5$ & $-56,0$ & $-54,5$ & $-28,5$ & $-22,5$ & $-21,0$ & $-20,0$ \\
\hline & 1,9 & $-72,5$ & $-71,0$ & $-69,5$ & $-68,0$ & $-66,5$ & $-65,0$ & $-63,5$ & $-62,0$ & $-60,0$ & $-30,5$ & $-23,5$ & $-21,5$ & $-20,5$ \\
\hline & 2,0 & $-80,0$ & $-78,5$ & $-76,5$ & $-75,0$ & $-73,5$ & $-72,0$ & $-70,0$ & $-68,0$ & $-66,5$ & $-33,0$ & $-24,5$ & $-22,5$ & $-21,0$ \\
\hline & 2,1 & $-88,0$ & $-86,5$ & $-84,5$ & $-83,0$ & $-81,0$ & $-79,0$ & $-77,0$ & $-75,0$ & $-72,5$ & $-35,5$ & $-26,0$ & $-23,5$ & $-21,5$ \\
\hline
\end{tabular}

Tabla 3. Reglas de inferencia para $T_{\mathrm{T}}$.

\begin{tabular}{|c|c|c|c|c|c|c|c|}
\hline & \multicolumn{6}{|c|}{ Temperatura inicial, $T_{i}\left({ }^{\circ} \mathrm{C}\right)$} \\
\hline & & $T_{i 5}$ & $T_{i 4}$ & $T_{i 3}$ & $T_{i 2}$ & $T_{i 1}$ & $T_{i 10}$ \\
\hline \multirow{10}{*}{ 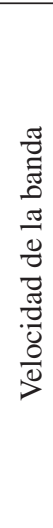 } & $\omega_{0}$ & $T_{\mathrm{t} 5}$ & $T_{\mathrm{t} 6}$ & $T_{\mathrm{t} 6}$ & $T_{\mathrm{t} 6}$ & $T_{\mathrm{t} 6}$ & $T_{\mathrm{t} 6}$ \\
\hline & $\omega_{1}$ & $T_{\mathrm{t} 5}$ & $T_{\mathrm{t} 5}$ & $T_{\mathrm{t} 5}$ & $T_{\mathrm{t} 5}$ & $T_{\mathrm{t} 6}$ & $T_{\mathrm{t} 6}$ \\
\hline & $\omega_{2}$ & $T_{\mathrm{t} 5}$ & $T_{\mathrm{t} 5}$ & $T_{\mathrm{t} 5}$ & $T_{\mathrm{t} 5}$ & $T_{\mathrm{t} 6}$ & $T_{\mathrm{t} 6}$ \\
\hline & $\omega_{3}$ & $T_{\mathrm{t} 4}$ & $T_{\mathrm{t} 4}$ & $T_{\mathrm{t} 4}$ & $T_{\mathrm{t} 5}$ & $T_{\mathrm{t} 6}$ & $T_{\mathrm{t} 6}$ \\
\hline & $\omega_{4}$ & $T_{\mathrm{t} 3}$ & $T_{\mathrm{t} 4}$ & $T_{\mathrm{t} 4}$ & $T_{\mathrm{t} 4}$ & $T_{\mathrm{t} 6}$ & $T_{\mathrm{t} 6}$ \\
\hline & $\omega_{5}$ & $T_{\mathrm{t} 3}$ & $T_{\mathrm{t} 3}$ & $T_{\mathrm{t} 3}$ & $T_{\mathrm{t} 3}$ & $T_{\mathrm{t} 6}$ & $T_{\mathrm{t} 6}$ \\
\hline & $\omega_{6}$ & $T_{\mathrm{t} 2}$ & $T_{\mathrm{t} 2}$ & $T_{\mathrm{t} 3}$ & $T_{\mathrm{t} 3}$ & $T_{\mathrm{t} 6}$ & $T_{\mathrm{t} 6}$ \\
\hline & $\omega_{7}$ & $T_{\mathrm{t} 1}$ & $T_{\mathrm{t} 2}$ & $T_{\mathrm{t} 2}$ & $T_{\mathrm{t} 2}$ & $T_{\mathrm{t} 5}$ & $T_{\mathrm{t} 6}$ \\
\hline & $\omega_{8}$ & $T_{\mathrm{t} 1}$ & $T_{\mathrm{t} 1}$ & $T_{\mathrm{t} 1}$ & $T_{\mathrm{t} 2}$ & $T_{\mathrm{t} 5}$ & $T_{\mathrm{t} 6}$ \\
\hline & $\omega_{9}$ & $T_{\mathrm{t} 0}$ & $T_{\mathrm{t} 0}$ & $T_{\mathrm{t} 1}$ & $T_{\mathrm{t} 1}$ & $T_{\mathrm{t} 5}$ & $T_{\mathrm{t} 6}$ \\
\hline
\end{tabular}

de valores de $T_{\mathrm{I}}$ y $\omega$, la diferencia existente entre dicha respuesta y el valor que aparece en la Tabla 2. Esta diferencia puede verse en la Tabla 4.

Los resultados obtenidos indican que la definición del controlador tipo Mamdani no es la más acorde para la aplicación objeto del presente trabajo, ya que, de implantarse este se estará incurriendo en consumos adicionales de nitrógeno líquido, debido principalmente al sobreenfriamiento de los productos a ser procesados, razón por la cual se hace necesario rediseñar el controlador presentado mediante la modificación de los conjuntos difusos.

\section{Rediseño del controlador difuso:}

Tomando como base los valores referenciales para la temperatura del túnel que aparecen en la Tabla 2 , se

Tabla 4. Desviaciones del control Mamdani $\left(T_{\mathrm{T}}\right)$ en función de $T_{\mathrm{i}} \mathrm{y} \omega$.

\begin{tabular}{|c|c|c|c|c|c|c|c|c|c|c|c|c|c|c|}
\hline & \multicolumn{13}{|c|}{ Temperatura inicial, $T_{i}\left({ }^{\circ} \mathrm{C}\right)$} \\
\hline & & 20,0 & 17,5 & 15,0 & 12,5 & 10,0 & $\mathbf{7 , 5 0}$ & 5,00 & 2,50 & $\mathbf{0}$ & -2.50 & $-5,00$ & $-7,50$ & $-10,0$ \\
\hline \multirow{10}{*}{ 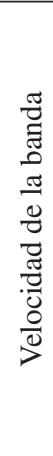 } & 1,2 & $1 \%$ & $3 \%$ & $4 \%$ & $6 \%$ & $8 \%$ & $10 \%$ & $12 \%$ & $-4 \%$ & $-2 \%$ & $32 \%$ & $32 \%$ & $32 \%$ & $32 \%$ \\
\hline & 1,3 & $-1 \%$ & $0 \%$ & $2 \%$ & $3 \%$ & $5 \%$ & $6 \%$ & $-2 \%$ & $-1 \%$ & $1 \%$ & $32 \%$ & $32 \%$ & $32 \%$ & $32 \%$ \\
\hline & 1,4 & $-1 \%$ & $0 \%$ & $1 \%$ & $4 \%$ & $5 \%$ & $7 \%$ & $10 \%$ & $-1 \%$ & $0 \%$ & $23 \%$ & $32 \%$ & $32 \%$ & $32 \%$ \\
\hline & 1,5 & $-2 \%$ & $-1 \%$ & $1 \%$ & $2 \%$ & $5 \%$ & $7 \%$ & $-2 \%$ & $0 \%$ & $1 \%$ & $15 \%$ & $32 \%$ & $32 \%$ & $32 \%$ \\
\hline & 1,6 & $-4 \%$ & $-3 \%$ & $-1 \%$ & $1 \%$ & $3 \%$ & $5 \%$ & $8 \%$ & $0 \%$ & $2 \%$ & $5 \%$ & $29 \%$ & $32 \%$ & $32 \%$ \\
\hline & 1,7 & $3 \%$ & $4 \%$ & $7 \%$ & $0 \%$ & $2 \%$ & $4 \%$ & $-3 \%$ & $-1 \%$ & $2 \%$ & $-1 \%$ & $23 \%$ & $29 \%$ & $32 \%$ \\
\hline & 1,8 & $-1 \%$ & $2 \%$ & $-5 \%$ & $-2 \%$ & $0 \%$ & $2 \%$ & $4 \%$ & $-2 \%$ & $1 \%$ & $10 \%$ & $39 \%$ & $25 \%$ & $32 \%$ \\
\hline & 1,9 & $-3 \%$ & $-1 \%$ & $1 \%$ & $3 \%$ & $5 \%$ & $0 \%$ & $-6 \%$ & $-3 \%$ & $0 \%$ & $15 \%$ & $33 \%$ & $23 \%$ & $29 \%$ \\
\hline & 2,0 & $-2 \%$ & $0 \%$ & $3 \%$ & $5 \%$ & $0 \%$ & $2 \%$ & $5 \%$ & $3 \%$ & $-2 \%$ & $6 \%$ & $28 \%$ & $17 \%$ & $25 \%$ \\
\hline & 2,1 & $-5 \%$ & $-3 \%$ & $-1 \%$ & $1 \%$ & $3 \%$ & $6 \%$ & $2 \%$ & $5 \%$ & $2 \%$ & $-1 \%$ & $21 \%$ & $12 \%$ & $23 \%$ \\
\hline
\end{tabular}


puede conseguir un conjunto de curvas que definen el comportamiento del valor de dicha temperatura en función de $\omega$, para un valor específico de temperatura inicial $T_{\mathrm{I}}$, como se muestra en la Figura 10.

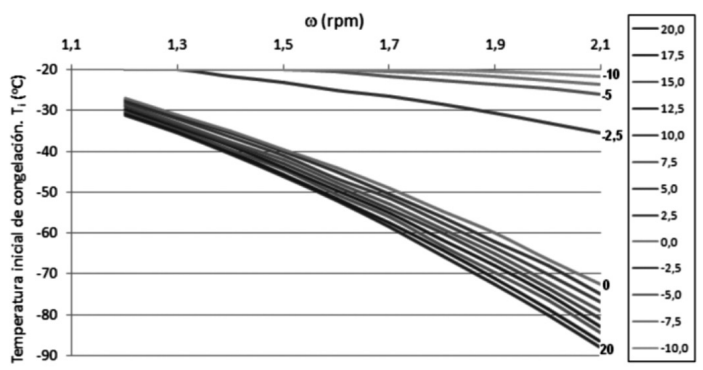

Figura 10.Comportamiento de $T_{\mathrm{T}}$ en función de $T_{\text {I }}$ y $\omega$.

Con las curvas de la Figura 10, se obtiene el conjunto de ecuaciones (10), que establece la dependencia de la temperatura del túnel $\left(T_{\mathrm{T}}\right)$ en función de la velocidad del rodillo de la banda transportadora $(\omega)$ para un valor específico de la temperatura inicial del producto $\left(T_{\mathrm{I}}\right)$. Con ello, se define la totalidad del conjunto difuso establecido para la temperatura inicial.

El sistema de ecuaciones descrito en las ecuaciones (10) servirá de fundamento para desarrollar las reglas difusas de un controlador tipo Takagi-Sugeno, de manera que:

Si $T_{i_{1}}>T_{i} \geq T_{i_{2}}$; entonces $T_{i_{2}} ;$ entonces $T_{T}$ es $f\left(T_{T\left(T_{i}\right)} ; \omega\right)$
En donde $f\left(T_{T\left(T_{i}\right)} ; \omega\right)$, es el resultado de interpolar los valores extremos de $T_{\mathrm{T}}$, del rango al cual pertenece $T_{\mathrm{I}}$.

$$
T_{T\left(T_{i}\right)}=\left\{\begin{array}{c}
f(-10 ; \omega)=57 \omega^{5}-47 \omega^{4}+1516 \omega^{3} \\
-2409 \omega^{2}+1891 \omega-607 \\
f(-7,5 ; \omega)=-7,39 \omega^{2}+20,74 \omega-34,4 \\
f(-5 ; \omega)=9,85 \omega^{2}+25,74 \omega-36,7 \\
f(-2,5 ; \omega)=-10,04 \omega^{2}+15,34 \omega-23,5 \\
f(0 ; \omega)=-16,29 \omega^{2}+3,27 \omega-7,6 \\
f(2,5 ; \omega)=17,61 \omega^{2}+5,46 \omega-8,7 \\
f(5 ; \omega)=17,61 \omega^{2}+3,79 \omega-7,2 \\
f(7,5 ; \omega)=17,99 \omega^{2}+3,53 \omega-7 \\
f(10 ; \omega)=19,13 \omega^{2}+5,73 \omega-8,5 \\
f(12,5 ; \omega)=-19,70 \omega^{2}+5,97 \omega-8,4 \\
f(15 ; \omega)=-19,51 \omega^{2}+4,04 \omega-6,8 \\
f(17,5 ; \omega)=-20,27 \omega^{2}+4,75 \omega-7 \\
f(20 ; \omega)=21,02 \omega^{2}+5,77 \omega-7,5
\end{array}\right\}
$$

Tal como se hizo con el controlador de tipo Mamdani, se procedió a obtener, para cada valor de $T_{\mathrm{I}} \mathrm{y} \omega$, los valores de salida del controlador para la temperatura del túnel, cuyas desviaciones respecto a los valores reales pueden verse en la Tabla 5.

De la Tabla 5 se puede asegurar que el controlador desarrollado mediante Takagi-Sugeno ofrece una mejor respuesta, ya que el porcentaje de desviación

Tabla 5. Desviaciones del control Takagi-Sugeno $\left(T_{\mathrm{T}}\right)$ en función de $T_{\mathrm{i}}$ y $\omega$.

\begin{tabular}{|c|c|c|c|c|c|c|c|c|c|c|c|c|c|c|}
\hline & \multicolumn{13}{|c|}{ Temperatura inicial, $T_{i}\left({ }^{\circ} \mathrm{C}\right)$} \\
\hline & & 20,0 & 17,5 & 15,0 & 12,5 & 10,0 & 7,50 & 5,00 & 2,50 & $\mathbf{0}$ & -2.50 & $-5,00$ & $-7,50$ & $-10,0$ \\
\hline \multirow{10}{*}{ 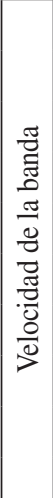 } & 1,2 & $-0,5 \%$ & $-0,1 \%$ & $0,1 \%$ & $0,3 \%$ & $0,6 \%$ & $0,6 \%$ & $0,0 \%$ & $0,0 \%$ & $0,5 \%$ & $-2,3 \%$ & $-0,5 \%$ & $0,7 \%$ & $-0,1 \%$ \\
\hline & 1,3 & $0,1 \%$ & $0,2 \%$ & $0,0 \%$ & $-0,2 \%$ & $-0,4 \%$ & $-0,5 \%$ & $0,1 \%$ & $-0,4 \%$ & $-0,4 \%$ & $2,7 \%$ & $-0,6 \%$ & $-0,4 \%$ & $-0,3 \%$ \\
\hline & 1,4 & $0,3 \%$ & $0,2 \%$ & $-0,3 \%$ & $0,4 \%$ & $-0,1 \%$ & $-0,5 \%$ & $-0,2 \%$ & $-0,2 \%$ & $-0,1 \%$ & $0,9 \%$ & $-0,2 \%$ & $-0,8 \%$ & $0,0 \%$ \\
\hline & 1,5 & $0,3 \%$ & $-0,1 \%$ & $0,3 \%$ & $-0,5 \%$ & $-0,1 \%$ & $0,5 \%$ & $0,3 \%$ & $0,4 \%$ & $-0,4 \%$ & $0,3 \%$ & $1,3 \%$ & $-0,4 \%$ & $0,0 \%$ \\
\hline & 1,6 & $0,2 \%$ & $-0,4 \%$ & $-0,5 \%$ & $-0,5 \%$ & $-0,4 \%$ & $-0,2 \%$ & $-0,6 \%$ & $0,1 \%$ & $0,2 \%$ & $-1,4 \%$ & $1,1 \%$ & $0,6 \%$ & $-0,3 \%$ \\
\hline & 1,7 & $-0,1 \%$ & $0,0 \%$ & $0,6 \%$ & $0,3 \%$ & $0,7 \%$ & $0,0 \%$ & $0,3 \%$ & $-0,3 \%$ & $0,2 \%$ & $-0,2 \%$ & $-0,5 \%$ & $0,0 \%$ & $-0,4 \%$ \\
\hline & 1,8 & $-0,4 \%$ & $0,2 \%$ & $-0,4 \%$ & $0,0 \%$ & $0,3 \%$ & $-0,1 \%$ & $-0,1 \%$ & $-0,1 \%$ & $0,0 \%$ & $-0,3 \%$ & $-1,0 \%$ & $0,0 \%$ & $0,3 \%$ \\
\hline & 1,9 & $-0,1 \%$ & $0,2 \%$ & $0,1 \%$ & $0,2 \%$ & $0,3 \%$ & $0,4 \%$ & $0,1 \%$ & $-0,1 \%$ & $0,3 \%$ & $0,3 \%$ & $-0,7 \%$ & $0,7 \%$ & $-0,4 \%$ \\
\hline & 2,0 & $0,1 \%$ & $0,1 \%$ & $0,3 \%$ & $0,3 \%$ & $0,1 \%$ & $-0,1 \%$ & $0,1 \%$ & $0,4 \%$ & $-0,4 \%$ & $-0,1 \%$ & $0,4 \%$ & $-0,1 \%$ & $-0,2 \%$ \\
\hline & 2,1 & $0,1 \%$ & $-0,1 \%$ & $-0,2 \%$ & $-0,3 \%$ & $-0,2 \%$ & $-0,1 \%$ & $-0,1 \%$ & $-0,1 \%$ & $0,1 \%$ & $0,2 \%$ & $0,3 \%$ & $-0,3 \%$ & $-0,2 \%$ \\
\hline
\end{tabular}


de los valores obtenidos de manera individual no supera el $3 \%$ en el peor de los casos, mientras que con el controlador Mamdani la desviación superaba en la mayoría de los casos el $5 \%$.

\section{EVALUACIÓN DEL SISTEMA PROPUESTO}

Una vez obtenidos los parámetros de entonación del controlador del lazo de velocidad de la banda y del controlador de temperatura del túnel, y establecida la configuración para el control del flujo másico y el diseño del control difuso de la temperatura, se interconectan los lazos de control tal como se ilustra en la Figura 11.

Después de configurada la simulación en el entorno LabView, se procede a la realización de un conjunto de pruebas, específicamente, cambio de las condiciones de operación y aplicación de perturbaciones, que permitan evaluar el comportamiento del sistema propuesto.

\section{Respuesta ante cambios en el valor de consigna del controlador de flujo másico}

Para esta prueba se consideraron las siguientes condiciones iniciales: a) Producto: churrascos de atún; b) $T_{\mathrm{I}}=10^{\circ} \mathrm{C}$, masa total $(M=200 \mathrm{~kg})$; c) Tiempo de simulación de $1000 \mathrm{~s}$.

El comportamiento del sistema en esta prueba se observa en la Figura 12, la que muestra que un
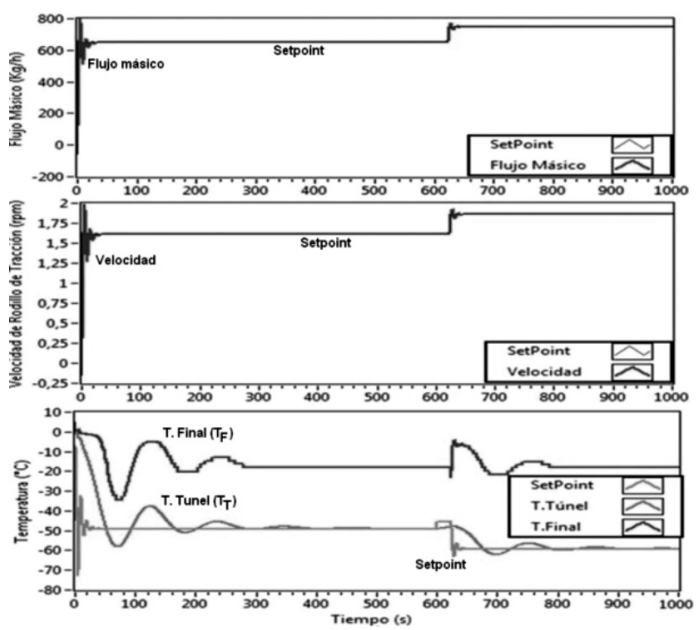

Figura 12. Respuesta del sistema ante cambios en el valor de consigna.

aumento en el flujo másico del alimento a congelar, desde $650 \mathrm{~kg} / \mathrm{h}$ hasta $700 \mathrm{~kg} / \mathrm{h}$, produce un aumento en la velocidad de rotación de giro del rodillo de tracción, desde 1,62 rpm hasta 1,75 rpm, lo que a su vez ocasiona que el controlador difuso disminuya la temperatura del túnel hasta $-60{ }^{\circ} \mathrm{C}$, logrando de esa manera que la temperatura final del alimento $\left(T_{\mathrm{F}}\right)$ esté alrededor de $\operatorname{los}-18^{\circ} \mathrm{C}$.

\section{Respuesta ante perturbaciones en la masa total} En este caso se mantuvieron las mismas condiciones iníciales de la primera prueba y fue aplicado un cambio

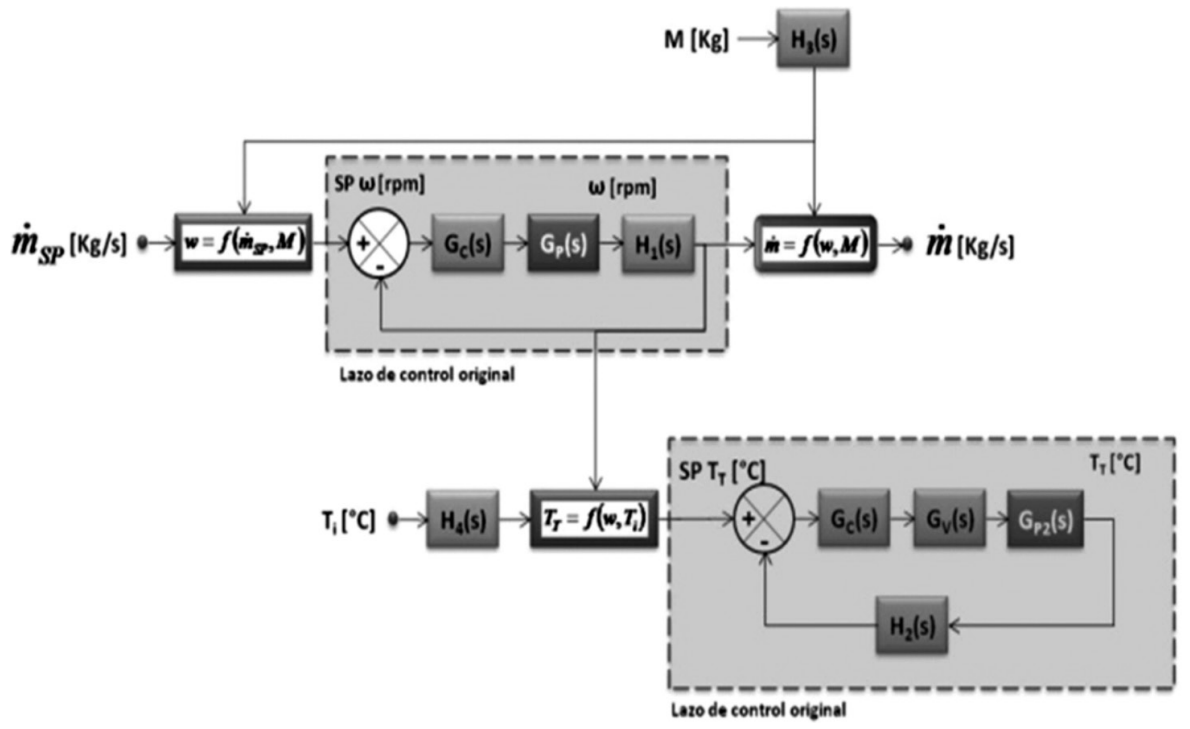

Figura 11. Diagrama en bloques del sistema de control completo. 
escalón positivo de $20 \mathrm{~kg}$ a los 600 segundos del tiempo de simulación (la masa de atún pasó de $200 \mathrm{~kg}$ a $220 \mathrm{~kg}$ ), obteniéndose los resultados presentados en la Figura 13, que permiten apreciar que un cambio en la masa del alimento a congelar tiene repercusiones directas en el comportamiento del lazo que controla la velocidad del rodillo de tracción de la banda transportadora (disminuye de 1,62 rpm a 1,47 rm), lo cual a su vez incrementa el tiempo de exposición del alimento dentro del túnel de congelación. En esas circunstancias, el controlador difuso reacciona modificando el valor de consigna de la temperatura del túnel, compensando dicha perturbación.

\section{Respuesta ante perturbaciones en la temperatura inicial}

Para esta prueba, las condiciones iniciales fueron las mismas que las de la primera prueba y se aplicó un cambio escalón de $+5^{\circ} \mathrm{C}$ a la temperatura inicial del churrasco de atún transcurridos 600 segundos del tiempo de simulación. La Figura 14, ilustra los resultados obtenidos en esta tercera prueba. En este

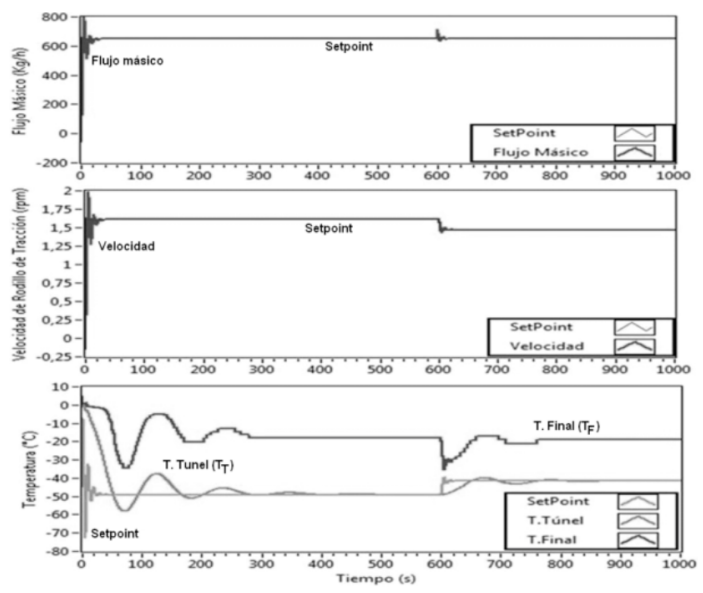

Figura 13. Respuesta del sistema ante perturbaciones en la masa total.

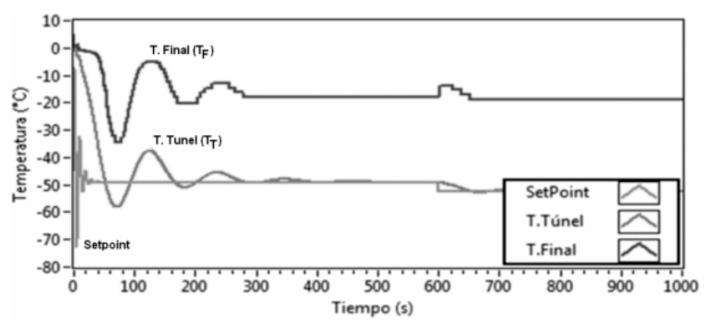

Figura 14. Respuesta del sistema ante perturbaciones en la temperatura inicial. caso, queda de manifiesto que cualquier perturbación en la temperatura de entrada del producto hace que el controlador difuso actúe para lograr mantener la temperatura de salida del alimento alrededor del valor deseado, es decir, $-18^{\circ} \mathrm{C}$.

\section{CONCLUSIONES}

Dadas las características del proceso de congelación de alimentos de la planta, donde los operadores participan activamente en el ajuste manual de los valores de consigna de la velocidad de la banda transportadora y la temperatura del túnel de congelamiento, son latentes las ventajas de la utilización de un controlador difuso. Esto último se verifica durante la fase de análisis de los resultados obtenidos al simular el comportamiento del sistema bajo la acción del controlador difuso propuesto en el presente trabajo.

En las pruebas preliminares se observa que los valores de las variables de salida no presentan grandes oscilaciones alrededor del valor de consigna. Esto se logró mediante la selección de los parámetros de entonación que permitieron obtener una respuesta rápida y con un sobrepico relativamente bajo.

El controlador desarrollado tipo Mamdani no produjo los resultados esperados, debido principalmente a la definición que se hizo de los conjuntos difusos, lo que obligó a rediseñar el controlador a ser implantado.

Por su parte, la respuesta del controlador desarrollado mediante los principios de Takagi-Sugeno arrojó una menor desviación con respecto a los valores deseados y fue seleccionado este por su facilidad de implantación (solo requiere de 13 reglas de inferencia versus las 66 que requirió el controlador de Mamdani).

Finalmente, de los resultados de las pruebas de simulación se puede inferir que el sistema propuesto basado en lógica difusa, permite obtener una respuesta rápida y estable cuando dicho sistema es sometido a diferentes perturbaciones.

\section{REFERENCIAS}

[1] E.C. Bellocq. "Deshidrocongelación de Zapallo Anco". Tesis para optar al grado de Magíster en Tecnología de Alimentos. 
Universidad Nacional Tecnológica. Buenos Aires, Argentina. 2012.

[2] A.I. Gómez, T.G. Cerón, V. Rodríguez y M.M. Vásquez. "Aspectos Tecnológicos de la Congelación en Alimentos". Temas Selectos de Ingeniería de Alimentos. Vol. 1 $\mathrm{N}^{\circ}$ 1, pp. 80-96. Agosto-Diciembre 2007. ISSN: $1547-1648$.

[3] R. De la Torre. "Congelación de Alimentos (Aspectos de Ingeniería)". 8 de agosto de 2015. URL: http://es.slideshare.net/alan robless/apuntesdecongelaciondealimentos

[4] Y. Choi and M.R. Okos. "Effects of Temperature and Composition on the Thermal Properties of Foods". Food Engineering and Process Applications. Elsevier Applied Science Publishers. London. 1986.

[5] R. Uporn and P. Luangpaiboon. "Cryogenic Freezing Process Optimization based on Desirability Function on the Path of Steepest Ascent". World Academy of Science, Engineering and Technology. Vol. $6 \mathrm{~N}^{\circ} 12$, pp. 1289-1294. December, 2012. ISSN: 1307-6892.

[6] S. Ramakrishnan, N. Gautam and R. Wysk. "A Tunnel Freezing Process with Adaptive Control - A Stochastic Modeling Approach". In 11th Annual Industrial Engineering Research Conference (IERC), Orlando, FL, United States of America, pp. 1-6. May, 2002.

[7] N. Shaikh and V. Prabhu. "Vision System for Model based Control of Cryogenic Tunnel Dreezers". Computers in Industry. Vol. 56, Issues 8-9, pp. 777-786. December, 2005. ISSN: 0166-3615. DOI: 10.1016/j. compind.2005.05.007

[8] N. Shaikh and V.Prabhu. "Model Predictive Controller for Cryogenic Tunnel Freezers". Journal of Food Engineering. Vol. $80 \mathrm{~N}^{\mathrm{o}} 2$, pp. 711-718. May, 2007. ISSN: 0260-8774. DOI: 10.1016/j.jfoodeng.2006.04.065

[9] V. Damian and C. Ciufudean. "Fuzzy Controller for Liquid Nitrogen Cryogenic Freezer". In the 12th WSEAS International Conference on Automatic Control, Modelling \& Simulation, pp. 201-206. Catania, Italy. May 2010.

[10] N. Shaikh and V. Prabhu. "Mathematical Modeling and Simulation of Cryogenic Tunnel Freezers". Journal of Food
Engineering. Vol. $80 \mathrm{~N}^{\mathrm{o}}$ 2, pp. 701-710. May, 2007. ISSN: 0260-8774. DOI: 10.1016/j. jfoodeng.2006.04.065

[11] J. Ramírez y B. León. "Predicción de Tiempos de Congelación y Descongelación de Alimentos". 15 de agosto de 2014. URL: http://www.agro-alimentarias.coop/ficheros/ doc/01292.pdf

[12] M. Frihat, B. Al-Zgoul, J. Radaedeh, M. Al-Radhdan and Al-Frihat. "Estimation of Food Product Freezing Time". Buletinul Institutului Politehnic Din Iaşi. Tomul LVIII (LXII), Fasc. 4, pp. 39-47. 2012.

[13] J. Madrid. "Refrigeración, Congelación y Envasado de Alimentos". AMV Ediciones Mundi-Prensa. Madrid, España. 2005.

[14] L. Otero, B. Guignon and P. Sanz Frutos. "Últimos Avances en Tecnologías de Congelación de Alimentos". Revista de Tecnología e Higiene de los Alimentos. No 440, pp. 82-89. 2013.

[15] S.E. Zorilla and A.C. Rubiolo. "Mathematical Modeling for Immersion Chilling and Freezing of Foods. Part I: Model Development". Journal of Food Engineering. Vol. $66 \mathrm{~N}^{\circ} 3$, pp. 329-338. February, 2005. ISSN: 02608774. DOI: $10.1016 /$ j.jfoodeng.2004.03.026

[16] M. Santos and A. Lespinard. "Numerical Simulation of Mushrooms During Freezing Using the FEM and an Enthalpy: Kirchhoff Formulation". International Journal of Heat and Mass Transfer. Vol. $47 \mathrm{~N}^{\circ} 12$, pp. 16711683. December, 2011. ISSN: 0947-7411. DOI: DOI 10.1007/s00231-011-0831-7

[17] Y.H. Tseng, C.C. Cheng, H.P. Cheng and D. Lee. "Novel Real-Time Diagnosis of the Freezing Process Using an Ultrasonic Transducer". Sensors. Vol. $15 \mathrm{~N}^{\circ} 15$, pp. 10332-10349. May, 2015. ISSN: 14248220. DOI: $10.3390 / \mathrm{s} 150510332$

[18] K. Ogata. "Modern Control Engineering". Fifth Edition. Prentice Hall. New Jersey, USA. 2010.

[19] J. Ziegler and N. Nichols. "Optimum Settings for Automatic Controllers". ASME Transactions. Vol. 64, pp. 759-768, November, 1942.

[20] V.M. Alfaro. "Identificación de Procesos Sobreamortiguados Utilizando Técnicas de Lazo Abierto y Lazo Cerrado". Epiciclos. Vol. $2 \mathrm{~N}^{\circ}$ 1, pp. 9-30. Noviembre 2003. ISSN: $1657-5636$. 
[21] P. Sourdillef and A. O'Dwyer. "Implementation of New Modified Smith Predictor Designs". In the Irish Signals and Systems Conference (ISSC 2004), Belfast, Northern Ireland, pp. 284-289. June-July, 2004.

[22] J. Collado. "Diseño de Estrategias de Control Predictivo Robusto basado en Intervalos Difusos para la Operación de Microrredes". Trabajo para optar al grado de Ingeniero Civil Eléctrico. Universidad de Chile. Santiago, Chile. 2013.

[23] Y. Misra and H.R. Kamath. "A Review on Application of Fuzzy Logic in Increasing of Efficiency of Industrial Process". International Journal of Latest Trends in Engineering and Technology (IJLTET). Vol. 1 N$^{\circ}$ 3, pp. 109-113. September, 2012. ISSN: 2278-621X.

[24] S. Revelo Andrade. "Simulación de un Convertidor Multinivel Apilable Controlado con Lógica Difusa". Tesis para optar al grado de Magíster en Ciencias con Especialidad en Ingeniería Electrónica. Universidad de las Américas Puebla. Puebla, México. 2006.
[25] G.W. Nurcahyo, S.M. Shamsuddin, R.A. Alias and M. Noor Md. Sap. "Selection of Defuzzification Method to Obtain Crisp Value for Representing Uncertain Data in a Modified Sweep Algorithm". Journal of Computer Science \& Technology (JCS\&T). Vol. $3 \mathrm{~N}^{\circ} 2$, pp. 22-28. October, 2003. ISSN: 1666-603.

[26] E. Mamdani and S. Assilian. "An Experiment in Linguistic Synthesis with a Fuzzy Logic Controller". International Journal ManMachine Studies. Vol. $7 \mathrm{~N}^{\circ}$ 1, pp. 1-13. January, 1975. ISSN: 1071-5819. DOI: 10.1016/S0020-7373(75)80002-2

[27] T. Takagi and M. Sugeno. "Fuzzy Identification of Systems and its Application to Modeling and Control". IEEE Transactions Systems, Man and Cybernetics. Vol. SMC-15 $\mathrm{N}^{\circ}$ 1, pp. 116-132. January-February, 1985. ISSN: 0018-9472. DOI: 10.1109/ TSMC.1985.6313399

[28] National Instruments. "LabVIEW System Identification Toolkit". 28 de agosto de 2015. URL: http://sine.ni.com/nips/cds/view/p/ lang/es/nid/209045 